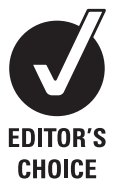

CHOICE
See editorial Commentary, p 475

${ }^{1}$ University Division of Anaesthesia, University of Cambridge, Cambridge, UK ${ }^{2}$ Wolfson Brain Imaging Centre, Department of Clinical Neurosciences, University of Cambridge, Cambridge, UK ${ }^{3}$ Department of Radiology, University of Cambridge, Addenbrooke's Hospital, Cambridge, UK

${ }^{4}$ Academic Neurosurgery Unit, Department of Clinical Neurosciences, University of Cambridge, Cambridge, UK

\section{Correspondence to} Dr Virginia Newcombe, University Division of Anaesthesia, University of Cambridge, Box 93 Addenbrooke's Hospital, Hills Road, Cambridge CB2 200, UK; vfjn2@wbic.cam.ac.uk

Received 29 September 2009 Revised 11 January 2010 Accepted 12 January 2010

\title{
Aetiological differences in neuroanatomy of the vegetative state: insights from diffusion tensor imaging and functional implications
}

\author{
Virginia F J Newcombe, ${ }^{1,2}$ Guy B Williams, ${ }^{2}$ Daniel Scoffings, ${ }^{3}$ Justin Cross, ${ }^{3}$ \\ T Adrian Carpenter, ${ }^{2}$ John D Pickard, ${ }^{2,4}$ David K Menon ${ }^{1,2}$
}

\begin{abstract}
Background An improved in vivo understanding of variations in neuropathology in the vegetative state (VS) may aid diagnosis, improve prognostication and help refine the selection of patients for particular treatment regimes. The authors have used diffusion tensor imaging (DTI) to characterise the extent and location of white matter loss in VS secondary to traumatic brain injury (TBI) and ischaemic-hypoxic injury.
\end{abstract}

Methods Twelve patients with VS (seven TBI, five ischaemic/hypoxic injuries) underwent MRI including DTI at a minimum of 3 months postinjury. Mean apparent diffusion coefficient, fractional anisotropy and eigenvalues were obtained for whole-brain grey and white matter, the pons, thalamus, ventral midbrain, dorsal midbrain and the corpus callosum. DTI measures of supratentorial damage were compared with a summed measure from the JFK modified Coma Recovery Scale (CRS-R) and with a three-point scale of functional magnetic resonance imaging (fMRI) response to an auditory paradigm to assess whether residual integrity of supratentorial white matter connectivity correlated with cortical processing.

Results Conventional radiological approaches did not detect lesions in regions where quantitative DTI demonstrated abnormalities. There was evidence of marked, broadly similar, abnormalities in the supratentorial grey- and white-matter compartments from both aetiologies. In contrast, discordant findings were found in the infratentorial compartment, with DTI abnormalities in the brainstem confined to the TBI group. Supratentorial DTI abnormalities correlated with the CRS$R$ as well as responses to an fMRI paradigm that detected convert cognitive processing.

Conclusions DTI may help to characterise differences in patients in VS. These findings may have implications for response to therapies, and should be taken into account in trials of interventions aimed at arousal in VS.

\section{INTRODUCTION}

The vegetative state (VS) encompasses a spectrum of patients who have emerged from coma to be awake, but show no indication of awareness. The aetiology of these clinical outcomes is varied and includes processes as disparate as traumatic brain injury (TBI), ischaemic/hypoxic injuries (IHI), intracerebral haemorrhage and infection. Modern imaging tools continue to provide more data on the neuroanatomical substrate of these conditions in vivo, and such information may be important to gain further insights into the disease process.
The commonest aetiologies of VS are TBI and IHI. While it is widely accepted that both these conditions arise from either damage to the brainstem/midbrain structures and/or diffuse axonal/ cortical damage, most data come from histopathological studies. Such studies show that thalamic damage is common in the vegetative state secondary to both IHI (neuronal loss secondary to hypoxia) and TBI (retrograde thalamic degeneration and/or neuronal loss secondary to hypoxia). ${ }^{12}$ While brainstem lesions have been recognised in IHI, these appear to affect predominantly brainstem grey matter nuclei, whereas more extensive white-matter damage is found in TBI (see Kinney and Samuels ${ }^{3}$ for an early review of histopathological studies). In TBI patients, focal lesions in the corpus callosum (diffuse axonal injuries (DAI) grade 2 ), or combined lesions in the corpus callosum and the dorsolateral brainstem (DAI grade 3), occurred in $71 \%$ of a series of 25 patients. ${ }^{1}$ In the same series, the majority of patients with IHI exhibited diffuse damage in the neocortex and hippocampus. ${ }^{1}$ Some of these patients also exhibited minor abnormalities in the brainstem, largely confined to the substantia nigra or other motor nuclei. These reports, using classical staining techniques, did not find extensive brainstem injuries for either aetiology. However, $\beta$ amyloid-precurser protein ( $\beta$-APP), a stain more sensitive to axonal injury, ${ }^{4}$ has been used in another series. ${ }^{5}$ This study, which focused on neuropathology in the pons, found axonal injury present in $80-100 \%$ of patients who survived more than $3 \mathrm{~h}$ after TBI. Axonal injury was also found in patients post-IHI, although it was noted that the intensity of $\beta$-APP accumulation was less than in cases of traumatic injury. ${ }^{5}$ These studies therefore suggest that classical neuropathological approaches may underestimate the extent and distribution of axonal injury, and that there may be subtle (but potentially important) differences in VS arising from different aetiologies. The ability to characterise ante mortem correlates of these pathological findings using modern imaging techniques has distinct advantages. A better understanding of the variations in neuropathology between patients and aetiologies could result in greater diagnostic precision and improved prognostication, and refine the selection and stratification of patients entered into clinical trial, in both the acute and chronic/rehabilitative stages.

Diffusion-weighted imaging is ideally placed to provide such insights into both the macroscopic and the microstructural damage found in VS. This technique characterises the natural displacements 
Table 1 Categorisation of functional magnetic resonance imaging (fMRI) responses to auditory stimuli $^{8} 9$

\begin{tabular}{|c|c|c|}
\hline Level & Description & fMRI responses \\
\hline Level 0 & $\begin{array}{l}\text { No cortical fMRI responses to any } \\
\text { auditory stimuli }\end{array}$ & None \\
\hline Level 1 & $\begin{array}{l}\text { Cortical responses characteristic of sound } \\
\text { perception only }\end{array}$ & $\begin{array}{l}\text { Activation of primary auditory cortex with } \\
\text { all auditory stimuli; no significant } \\
\text { differences in } \mathrm{fMRI} \text { activation patterns } \\
\text { between signal correlated noise, words } \\
\text { and ambiguous versus non-ambiguous } \\
\text { words }\end{array}$ \\
\hline Level 2 & $\begin{array}{l}\text { Distinction of words versus signal } \\
\text { correlated noise }\end{array}$ & $\begin{array}{l}\text { Activation of primary auditory cortex with } \\
\text { all auditory stimuli; significant differences } \\
\text { in fMRI activation patterns between signal } \\
\text { correlated noise and words (bilateral } \\
\text { activation centred on the superior } \\
\text { temporal sulcus }{ }^{8} \text { as well as a left- } \\
\text { lateralized response in the left inferior } \\
\text { frontal gyrus; no significant differences in } \\
\text { fMRI activation patterns between } \\
\text { sentences containing ambiguous and non- } \\
\text { ambiguous words }\end{array}$ \\
\hline Level 3 & $\begin{array}{l}\text { Distinction of words versus signal corre- } \\
\text { lated noise } \\
\text { Distinction of ambiguous from unambig- } \\
\text { uous words }\end{array}$ & $\begin{array}{l}\text { Activation of primary auditory cortex with } \\
\text { all auditory stimuli; significant differences } \\
\text { in fMRI activation patterns between signal } \\
\text { correlated noise and words (bilateral } \\
\text { activation centred on the superior } \\
\text { temporal sulcus }{ }^{8} \text { as well as a left- } \\
\text { lateralised response in the left inferior } \\
\text { frontal gyrus; additional significant } \\
\text { differences in fMRI activation patterns } \\
\text { between sentences containing ambiguous } \\
\text { and non-ambiguous words (left and right } \\
\text { inferior frontal gyrus (pars triangularis), } \\
\text { and a region of the left posterior inferior } \\
\text { temporal cortex) }\end{array}$ \\
\hline
\end{tabular}

of water molecules. The diffusion tensor can be used to represent the magnitude of water diffusion (apparent diffusion coefficient, $\mathrm{ADC}$ ), how directional the diffusion is (anisotropy) and the orientation of that direction (eigenvectors/eigenvalues). The diffusion process may be affected by various tissue structures and environments, including pathological processes such as demyelination, axonal truncation or microglia activation. In this study, we used diffusion tensor imaging to characterise and differentiate the extent and location of white-matter loss in patients with disorders of consciousness secondary to traumatic brain injury and ischaemic-hypoxic injury.

\section{METHODS}

These studies were conducted on a bank of data from patients with VS, which have been used for a number of behavioural and imaging analyses. The data in this manuscript are based on 12 (seven TBI, five IHI), who underwent $M R$ imaging at a minimum of 3 months postinjury using a $3 \mathrm{~T}$ Siemens Magnetom Total Imaging Matrix (TIM) Trio system. Patients were characterised as VS based on a full assessment using the JFK modified Coma Recovery Scale (CRS-R). ${ }^{6}$ Patients included in this analysis were selected primarily on the basis of DTI data quality, and no dataset was excluded once initial DTI processing revealed data of adequate quality. Images were usually excluded on the basis of excessive movements. In order to avoid bias, all such exclusions preceded placement of regions of interest and measurement of DTI variables. Informed assent for acquisition of imaging data for research was obtained from the next of kin in all cases. Ethical approval was obtained from the Local Research Ethics Committee. Thirty-two age-matched controls (healthy volunteers) underwent an identical imaging protocol which included a 3D T1 weighted structural sequence, magnetisation prepared rapid gradient echo (MPRAGE), functional magnetic resonance imaging (fMRI) and diffusion tensor
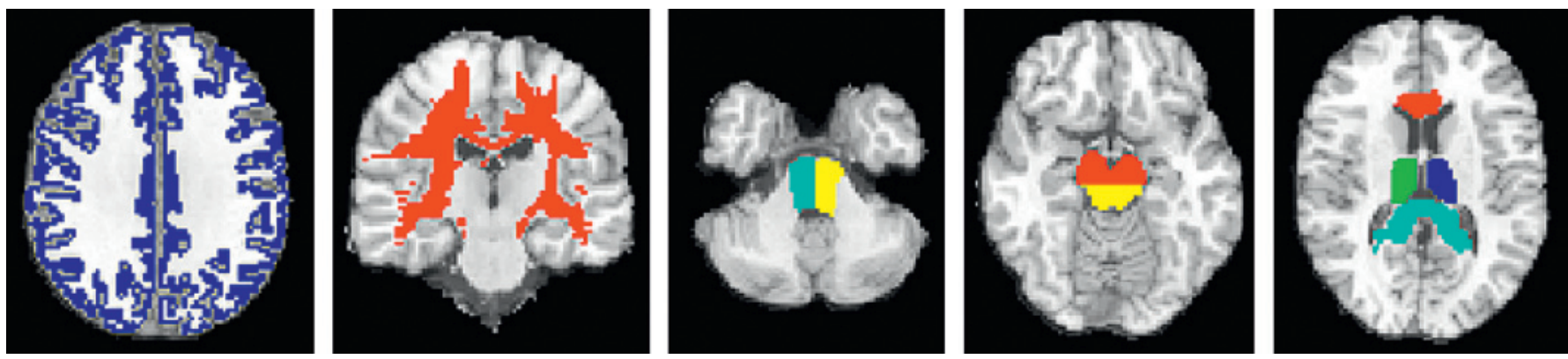

Figure 1 Examples of regions of interest used. From left to right, whole-brain grey matter, supratentorial white matter, the pons (left and right); midbrain (anterior and posterior); and thalamus, anterior corpus callosum, and posterior corpus callosum respectively. 
Table 2 Demographic and clinical characteristics of the study subjects

\begin{tabular}{|c|c|c|c|c|c|c|}
\hline Patient & Gender & $\begin{array}{l}\text { Age at } \\
\text { injury }\end{array}$ & Aetiology & $\begin{array}{l}\text { Time to scan } \\
\text { after injury (days) }\end{array}$ & $\begin{array}{l}\text { JFK Coma Recovery } \\
\text { Scale-Revised }^{6}\end{array}$ & $\begin{array}{l}\text { Functional magnetic resonance } \\
\text { imaging language }\end{array}$ \\
\hline 1 & $\mathrm{~F}$ & 49 & IHI & 209 & 7 & 1 \\
\hline 2 & $M$ & 33 & IHI & 325 & 6 & 2 \\
\hline 3 & $\mathrm{~F}$ & 38 & IHI & 1518 & 7 & 1 \\
\hline 4 & $\mathrm{~F}$ & 47 & IHI & 509 & 7 & 1 \\
\hline 5 & $\mathrm{~F}$ & 41 & IHI & 280 & 8 & 1 \\
\hline 6 & $M$ & 58 & Assault & 176 & 7 & 3 \\
\hline 7 & $M$ & 21 & Assault & 175 & 6 & 3 \\
\hline 8 & $M$ & 64 & Fall & 198 & 9 & 3 \\
\hline 9 & $\mathrm{~F}$ & 37 & Fall & 681 & 5 & 1 \\
\hline 10 & $M$ & 20 & RTA & 578 & 9 & 1 \\
\hline 11 & $\mathrm{~F}$ & 36 & RTA & 105 & 9 & 3 \\
\hline 12 & $M$ & 66 & RTA & 435 & 5 & 1 \\
\hline
\end{tabular}

*Level 1, response to sound only in the auditory cortex; level 2, response to speech; level 3, retrieval of semantic information. $\mathrm{F}$, female; IHI, ischaemic/hypoxic injuries; M, male; RTA, road traffic accident.

imaging. The large size compared with the patient population was due to two main reasons: first, the patient group is a difficult group to image successfully, and as DTI parameters in controls are well known to have great variability, it was decided to make the group large to account for this. The DTI parameters were as follows; 12 non-collinear directions, $5 \mathrm{~b}$ values ranging from 338 to $1588 \mathrm{~s} / \mathrm{mm}^{2}, 5 \mathrm{~b}=0$ images, field of view $192 \mathrm{~mm} \times 192 \mathrm{~mm}$, acquisition matrix 96×96, 63 axial slices, $2 \mathrm{~mm}$ slice thickness, TR=8300 ms, TE=98 ms. All images were inspected to ensure that movement during the scan was within acceptable limits $(<4 \mathrm{~mm})$. Patients were also scanned with conventional MR sequences (proton density and T2-weighted spin echo (PD-T2), gradient echo (GE), fluid attenuated inversion recovery (FLAIR)) and these were inspected by two neuroradiologists (JC and DS). The presence of lesions that may confound the study was noted (table 1). None of the patients had ventricular shunts, cranioplasty plates or any other metallic implants that would cause artefacts.

Regions of interest (ROIs) were manually drawn using Analyze 7.0 (Bioimaging Imaging Resource, Rochester, Minnesota, USA) ${ }^{10}$ in MNI125 space using Colin $27^{11}$ as a high-resolution, high-signalto-noise template and included the pons, ventral midbrain, dorsal midbrain, thalamus and corpus callosum (genu and splenium) (figure 1). A supratentorial whole-brain central white matter (WBCWM) ROI was created by subtracting the cortical grey matter, the transitional zone and infratentorial WM from a wholebrain white-matter mask segmented using FMRIB's Automated Segmentation Tool (FAST). ${ }^{12}$ The segmentation also produced a whole-brain grey matter mask (WBGM). These ROIs were transformed into the normalised space (see below for methods). All

Table 3 Conventional imaging characteristics of the patients with percentage of patients showing lesions in the sites shown

\begin{tabular}{llll}
\hline & & $\begin{array}{l}\text { Traumatic brain injury } \\
\mathbf{n}(\%)\end{array}$ & $\begin{array}{l}\text { Ischaemic-hypoxic injury } \\
\mathbf{n}(\%)\end{array}$ \\
\hline Supratentorial white matter & Corpus callosum & $3(43)$ & 0 \\
& Corona radiata & $1(14)$ & $1(20)$ \\
& Lobar white matter & $3(43)$ & $2(40)$ \\
& Internal capsule & 0 & 0 \\
Cortical contusions & Frontal & $2(29)$ & 0 \\
& Temporal & $2(29)$ & 0 \\
& Parietal & 0 & 0 \\
Cortical microhaemorrhages & Occipital & $1(14)$ & 0 \\
& Frontal & $1(14)$ & 0 \\
& Temporal & $2(29)$ & 0 \\
Subcortical grey matter lesions & Parietal & 9 & 0 \\
& Occipital & 0 & 0 \\
& Thalamus & $2(29)$ & 0 \\
& Basal ganglia & $2(29)$ & $2(40)$ \\
& Dorsolateral midbrain & $4(57)$ & $1(20)$ \\
& Ventrolateral brainstem & $2(29)$ & $1(20)$ \\
& Cerebral peduncles & $3(43)$ & 0 \\
& Pons & $2(29)$ & $1(20)$ \\
& Medulla & $3(43)$ & 0 \\
& Cerebellum & $2(29)$ & $1(20)$ \\
& Ventricular enlargement & $4(57)$ & $3(60)$ \\
& Cortical & $2(29)$ & $5(100)$ \\
& White matter & $1(14)$ & $2(40)$ \\
& Brainstem & $2(29)$ & $2(40)$ \\
& Thalamic/basal ganglia & $1(14)$ & $2(40)$ \\
\hline \multirow{5}{*}{ and cerebellar lesions } & &
\end{tabular}


Table 4 Conventional imaging characteristics of the patients and abnormalities detected using diffusion tensor imaging

\begin{tabular}{|c|c|c|c|c|c|c|}
\hline & $\begin{array}{l}\text { Conventional } \\
\text { TBI } \\
\text { n }(\%)\end{array}$ & $\begin{array}{l}\text { IHI } \\
\text { n (\%) }\end{array}$ & $\begin{array}{l}\text { Fractional anisotropy } \\
\text { TBI } \\
\text { n (\%) }\end{array}$ & $\begin{array}{l}\text { IHI } \\
\text { n (\%) }\end{array}$ & $\begin{array}{l}\text { Apparent diffusion coefficient } \\
\text { TBI } \\
\text { n (\%) }\end{array}$ & $\begin{array}{l}\text { IHI } \\
\text { n (\%) }\end{array}$ \\
\hline Whole-brain central white matter & $1(14)$ & $2(40)$ & $7(100)$ & $5(100)$ & $7(100)$ & $5(100)$ \\
\hline Anterior corpus callosum & 0 & 0 & $6(86)$ & $4(80)$ & $6(86)$ & $3(60)$ \\
\hline Posterior corpus callosum & $3(43)$ & 0 & $7(100)$ & $5(100)$ & $7(100)$ & $5(100)$ \\
\hline Thalamus & $2(29)$ & 0 & 1 (14) & $5(100)$ & $6(86)$ & $3(60)$ \\
\hline Pons & $2(29)$ & $1(20)$ & $6(86)$ & 0 & $7(100)$ & $1(20)$ \\
\hline
\end{tabular}

Numbers of patients with lesions in the areas qualitatively seen are compared with the number of patients with abnormalities detected using diffusion tensor imaging in normal appearing tissue.

Parameters more than 2 standard deviations from the control mean in the regions of interest analysed were considered damaged.

$\mathrm{IHI}$, ischaemic/hypoxic injuries; $\mathrm{TBI}$, traumatic brain injuries.

coregistered images were visually inspected to ensure that ROIs corresponded to the regions specified. Manual adjustment was performed using Analyze $7.0^{10}$ if they did not. Care was taken to ensure that the ROIs did not contain any lesions, as blood breakdown products such as haemosiderin cause signal drop-out with DTI sequences. Thus, all ROIs contained only tissue that appeared 'normal' on conventional MR. Any misclassified tissue in the WBCWM and WMGM masks was manually removed using Analyze 7.0. ${ }^{10}$ The mean ADC, fractional anisotropy (FA) and eigenvalues for the different ROIs were calculated. Such an ROIbased approached was chosen over voxel-based methods including tract-based spatial statistics, ${ }^{13}$ as the often marked atrophy of the brains which can cause coregistration difficulties meant that it was important to be able to manually adjust the regions to ensure like was compared with like.

The DTI data underwent eddy current correction, and FA, $\mathrm{ADC}$ and eigenvalue maps were created using the FMRIB's Diffusion Toolbox (FDT) ${ }^{14}$ To aid coregistration, the skull and extracranial soft tissue were stripped from the MPRAGE images using the Brain Extraction Tool. ${ }^{15}$ The diffusion-weighted data were normalised using a two-step approach. First, all patient and control MPRAGE images were coregistered to the MNI152 template using the vtkCISG normalised mutual information algorithm. ${ }^{16}$ The $b=0$ image was subsequently coregistered to the subject's own MPRAGE. The transformation matrix normalising the MPRAGE image was then applied to the $b=0$ image.

DTIquery ${ }^{17}$ was used in the subject's native space to create whole-brain tractography using the FACT algorithm, ${ }^{18}$ a variablestep streamline tracking method. Seed points for tracts were placed evenly throughout the brain as a grid with spacing of $4 \mathrm{~mm}$. The tractography parameters were: a step-size of $2 \mathrm{~mm}$, deflection angle of $45^{\circ}$ and FA termination threshold of 0.15 . Using the supratentorial WBCWM mask, to reduce errors due to noise, the number of 'tracts' greater than $3 \mathrm{~cm}$ were counted to create a surrogate marker of the burden of white matter injury.

In order to assess the clinical relevance of the DTI parameters, we compared them with a summary measure of functional status - the summed highest score derived from the JFK CRS-R. ${ }^{67}$ We also wished to explore whether the residual integrity of supratentorial white matter connectivity correlated with
Figure 2 Fluid-attenuated inversion recovery (FLAIR) and 'global' tractography with representative examples from the control, traumatic brain injury (TBI) and

ischaemic-hypoxic injury (IHI) groups providing a qualitative image of white matter integrity. This approach is illustrative, and was not used to quantify diffusion tensor imaging (DTI) parameters. It can be clearly seen that the amount of 'paths' able to be traced is far less in the patients than the controls. Despite relatively unremarkable appearances on conventional structural imaging (see TBI patient above) the DTI images showed consistent and striking loss of white matter. Also note the preservation of brainstem white matter tracts in $\mathrm{IHI}$ when compared with TBI (see arrows; the brainstem in the control subject is visualised despite the presence of a more substantial overlapping temporal lobe). For ease of visualisation, tracts with lengths less than $3 \mathrm{~cm}$ are not shown, and the coronal images are overlaid on fractional anisotropy maps.

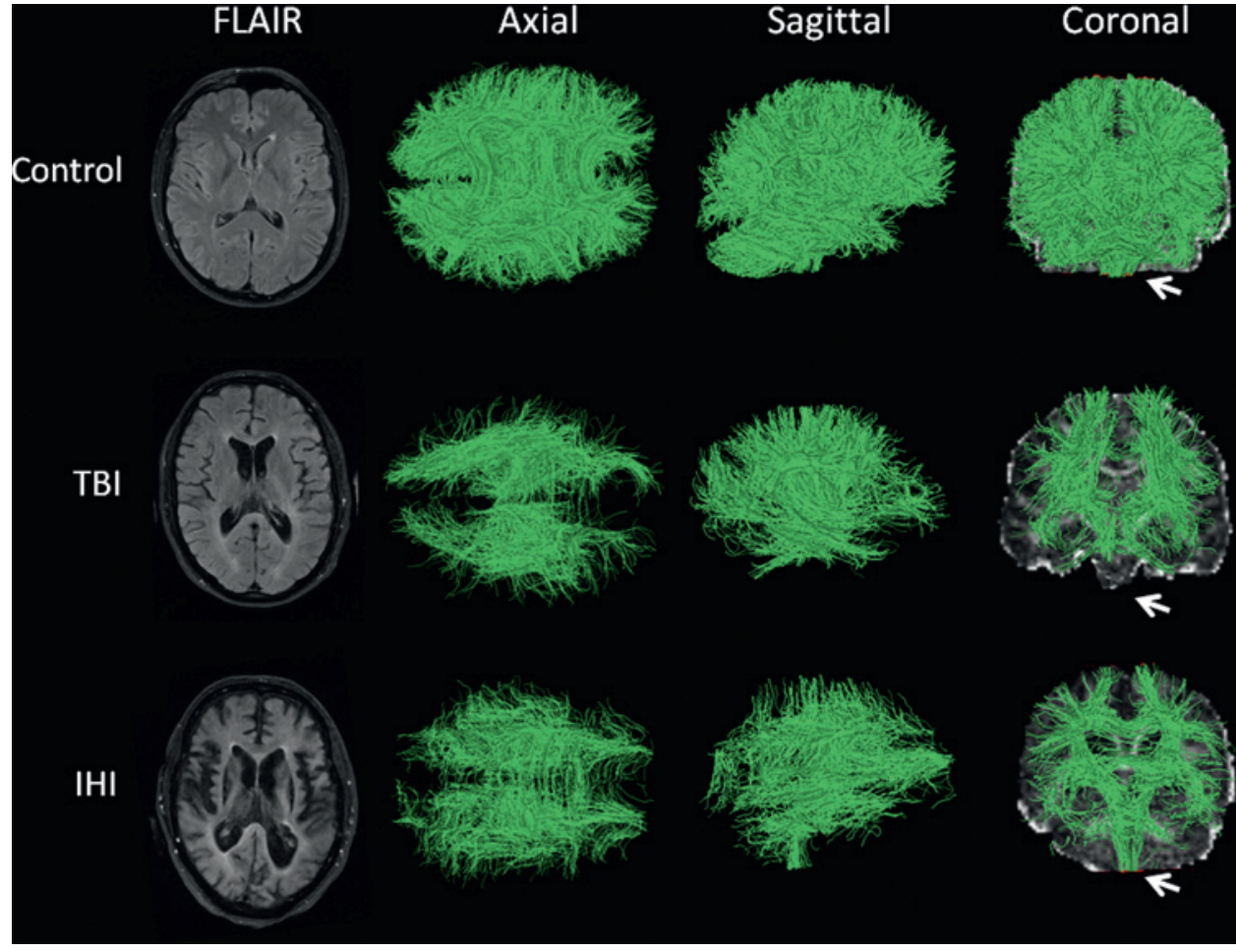


Table 5 Comparison of patients with vegetative state secondary to hypoxic brain injury to those caused by traumatic brain injury and controls

\begin{tabular}{|c|c|c|c|c|c|}
\hline & & & $\begin{array}{l}\text { Controls } \\
\text { Median (IOR) } \\
\end{array}$ & $\begin{array}{l}\text { Traumatic brain injury } \\
\text { Median (IOR) }\end{array}$ & $\begin{array}{l}\text { Hypoxic brain injury } \\
\text { Median (IQR) }\end{array}$ \\
\hline \multirow{4}{*}{$\begin{array}{l}\text { Whole-brain central white matter } \\
\text { (WBCWM) }\end{array}$} & & FA & $0.42(0.41$ to 0.43$)$ & $0.25(0.20 \text { to } 0.32)^{*}$ & $0.27(0.22 \text { to } 0.28)^{*}$ \\
\hline & & $A D C$ & $0.66(0.65$ to 0.67$)$ & $1.13(0.90 \text { to } 1.28)^{*}$ & $1.21(1.02 \text { to } 1.34)^{*}$ \\
\hline & & Radial & $0.50(0.49$ to 0.52$)$ & $1.03(0.74 \text { to } 1.12)^{*}$ & $1.06(0.89 \text { to } 1.44)^{*}$ \\
\hline & & Tracts & 42367 (3856 to 5109 ) & $1225(782 \text { to } 2042)^{*}$ & $748(173 \text { to } 3135)^{*}$ \\
\hline \multirow[t]{3}{*}{ Whole-brain grey matter } & & FA & 1.73 (1.68 to 1.81$)$ & 0.17 (0.13 to 0.19$)$ & $0.17(0.15$ to 0.20$)$ \\
\hline & & Radial & $0.73(0.71$ to 0.75$)$ & $0.84(0.71$ to 0.87$)$ & $1.06(0.82 \text { to } 1.06)^{*}$ \\
\hline & & Tracts & 4517 (4127 to 5611$)$ & 1112 (663 to 2001) & 720 (158 to 3019$)^{*}$ \\
\hline \multirow[t]{6}{*}{ Cortical contusions } & Anterior & FA & $0.57(0.52$ to 0.59$)$ & $0.33(0.21 \text { to } 0.48)^{*}$ & $0.45(0.28 \text { to } 0.51)^{*}$ \\
\hline & & $A D C$ & 0.69 (0.67 to 0.72$)$ & $0.99(0.85 \text { to } 1.19)^{*}$ & $0.86(0.75 \text { to } 1.28)^{*}$ \\
\hline & & Axial & 1.17 (1.14 to 1.24$)$ & $1.37(1.19 \text { to } 1.57)^{*}$ & 1.34 (1.19 to 1.62$)$ \\
\hline & & Radial & 0.44 (0.41 to 0.48 ) & $0.88(0.59 \text { to } 1.06)^{*}$ & $0.62(0.53 \text { to } 1.62)^{*}$ \\
\hline & & Radial & 0.36 (0.32 to 0.39 ) & $0.74(0.68 \text { to } 0.78)^{*}$ & $0.87(0.78 \text { to } 1.36)^{*}$ \\
\hline & & Tracts & 319 (224 to 443$)$ & $22(0 \text { to } 56)^{*}$ & $17(3 \text { to } 34)^{*}$ \\
\hline \multirow[t]{5}{*}{ Thalamus } & & FA & 0.34 (0.33 to 0.36$)$ & $0.32(0.30$ to 0.35$)$ & $0.42(0.40 \text { to } 0.47)^{*} \dagger$ \\
\hline & & $A D C$ & $0.66(0.63$ to 0.68$)$ & $0.85(0.83 \text { to } 0.88)^{*}$ & $0.79(0.67 \text { to } 0.92)^{*}$ \\
\hline & & Axial & 0.90 (0.88 to 0.93$)$ & $1.12(1.07 \text { to } 1.17)^{*}$ & $1.01(0.95 \text { to } 1.28)^{*}$ \\
\hline & & Radial & $0.54(0.52$ to 0.56$)$ & $0.72(0.67 \text { to } 0.74)^{*}$ & 0.58 (0.51 to 0.73$)$ \\
\hline & & Tracts & 728 (611 to 825$)$ & $212(137 \text { to } 318)^{*}$ & $86(28 \text { to } 497)^{*}$ \\
\hline \multirow[t]{5}{*}{ Midbrain } & Ventral & FA & 0.56 (0.54 to 0.59$)$ & $0.39(0.36 \text { to } 0.48)^{*}$ & $0.59(0.56$ to 0.60$) \dagger$ \\
\hline & & $A D C$ & 0.61 (0.57 to 0.64 ) & $0.69(0.63 \text { to } 0.80)^{*}$ & 0.67 (0.58 to 0.70$)$ \\
\hline & & Axial & $1.02(0.98$ to 1.09$)$ & 0.97 (0.89 to 1.25$)$ & $1.09(0.95$ to 1.16$)$ \\
\hline & & Radial & 0.40 (0.37 to 0.42 ) & $0.55(0.50 \text { to } 0.63)^{*}$ & $0.43(0.39$ to 0.48$) \dagger$ \\
\hline & & Tracts & 618 (563 to 753$)$ & $74(10 \text { to } 499)^{*}$ & $133(50 \text { to } 260)^{*} \dagger$ \\
\hline \multirow{3}{*}{ Pons } & & Axial & 0.93 (0.89 to 0.97$)$ & $1.01(0.94 \text { to } 1.08)^{*}$ & $0.97(0.86$ to 1.06$)$ \\
\hline & & Radial & $0.41(0.39$ to 0.43$)$ & $0.56(0.54 \text { to } 0.61)^{*}$ & $0.43(0.39$ to 0.48$) \dagger$ \\
\hline & & Tracts & 347 (263 to 407$)$ & $60(30 \text { to } 238)^{*}$ & $87(12 \text { to } 157)^{*} \dagger$ \\
\hline
\end{tabular}

The median (IQR) values for the traumatic brain injury and controls are listed in tables 3 , 4. Significant comparisons $(p<0.017)$ are shown.

* Significant differences of each patient group from the controls.

†Significant differences between the two patient groups.

$A D C$, apparent diffusion coefficient; FA, fractional anisotropy.

cortical processing as demonstrated by fMRI, independent of the ability to demonstrate behavioural outputs. We therefore compared DTI measures of supratentorial damage with a fourpoint scale of fMRI responses with an auditory fMRI paradigm based on a hierarchical pattern of speech processing described in Rodd et al, ${ }^{19}$ and previously described in MCS and VS by Coleman et $a l^{8}{ }^{9}$ (see table 1) with level 0 being no cortical fMRI response to auditory stimuli; level 1 , activations characteristic of response to sound only; level 2 , including activations characteristic of response to speech; and level 3 retrieval of semantic information. Statistical analyses were conducted using SPSS (SPSS 14.0; SPSS, Chicago, Illinois), and graphs were produced using StatView. Due to the small sample size in the HIH group all analyses, used non-parametric statistics. Mann-Whitney U (MHU) was used for unpaired tests, Spearman $\rho$ was used for correlations, and the Jonckheere-Terpstra test was used to test for trend. Statistical significance was accepted if the $p$ values were $<0.05$, corrected for multiple comparisons between the three groups (ie, $\mathrm{p}<0.017$ was considered significant).

\section{RESULTS}

Five patients (four female, median age 41 (range 33-49) years) with ischaemic brain injuries were compared with seven TBI (two female, median age 39 (range 21-67) years) (table 2). All were classified as VS according to the CRS-R. These patients were compared with 32 controls (seven female, median age 25.2 (range 25-70) years). No significant differences were found between groups for age $(p=0.536)$, or in the time to scan from ictus in the patient populations (IHI median 325 (range 209-1518) days, TBI median 198 (range 105-681) days, $\mathrm{p}=0.172$ ). Table 3 shows the radiological findings on structural MRI sequences (MPRAGE, PDT2, FLAIR and gradient echo sequences) in the patient group. It is important to note that DTI was more sensitive than conventional radiology-where 
Figure 3 Fractional anisotropy values for the regions of interest studied. The central lines in the boxes denote the median values, the upper and lower edges the 75th and 25 th percentiles, the error bars the 90th and 10th percentiles, and the closed circles the data outside these percentiles. ${ }^{*} \mathrm{p}<0.017$; ${ }^{* *}$ $\mathrm{p}<0.01 ;{ }^{* *} \mathrm{p}<0.001$. IHI, ischaemic/ hypoxic injuries; NS, non-significant; $\mathrm{TBI}$, traumatic brain injuries; WBCWM, whole-brain central white matter; WBGM, whole-brain grey matter.
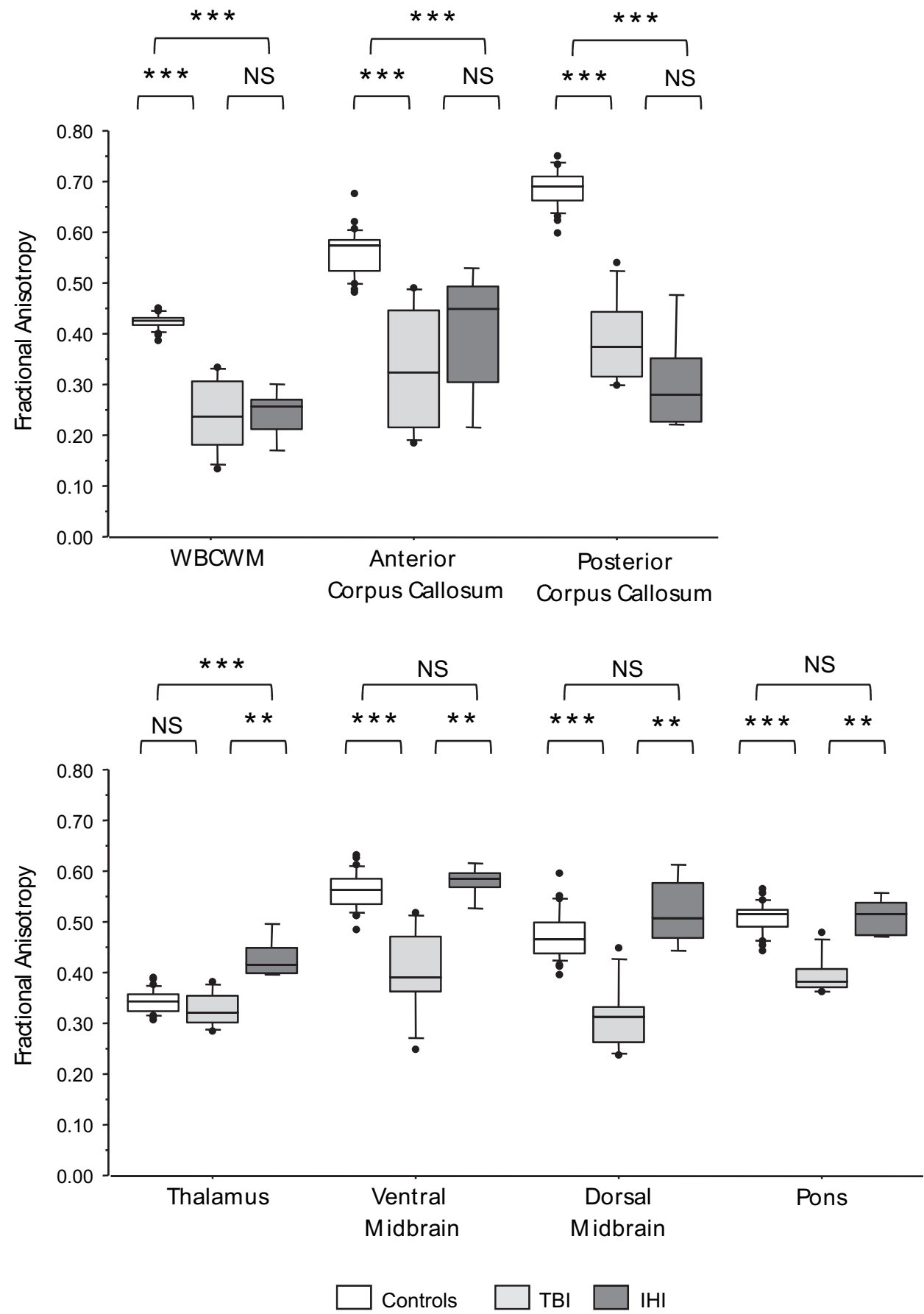

quantitative DTI measures were calculated, these consistently demonstrated abnormalities in regions where no lesions were detectable by experienced neuroradiologists (tables 3, 4).

Figure 2 shows whole-brain tractography from a representative control subject, TBI patient and IHI patient. These qualitative appearances were underpinned by quantitative measures of the DTI parameters FA and ADC as well as 'track counts' in both supratentorial and infratentorial structures derived from the source DTI images (table 5). It is important to note that the lack of brainstem visualisation was not due to a lack of MR coverage of this area, but the consequence of a decrease in FA, which meant that the tracts could not be traced using standard tracking parameters. $\mathrm{ADC}$ and FA were compared within each
ROI for males and females in the control group ADC, and FA values for left and right sides were compared for all ROIs in both the control and patient groups separately. No significant differences were found for any of these comparisons, and for subsequent analyses, data from right and left sides were combined.

In both patient groups, FA was significantly decreased and $\mathrm{ADC}$ increased compared with controls in the whole-brain white matter, anterior corpus callosum and posterior corpus callosum (figures 3, 4). Both patient groups had higher ADCs than controls in the WBGM and thalamic ROIs. DTI measures were broadly similar for the two aetiologies in these supratentorial ROIs. However, the TBI patients showed significantly lower FA values than both the control group and the IHI in the 
Figure 4 Apparent diffusion coefficient (ADC) values for the regions of interest studied. The central lines in the boxes lower edges the 75th and 25th percentiles, the error bars the 90th and 10th percentiles, and the closed circles the data outside these percentiles. ${ }^{*} \mathrm{p}<0.017$; ${ }^{* *} \mathrm{p}<0.01$; ${ }^{* * *} \mathrm{p}<0.001$. IHI, ischaemic/hypoxic injuries; NS, nonsignificant; TBI, traumatic brain injuries; WBCWM, whole-brain central white matter; WBGM, whole-brain grey matter. denote the median values, the upper and
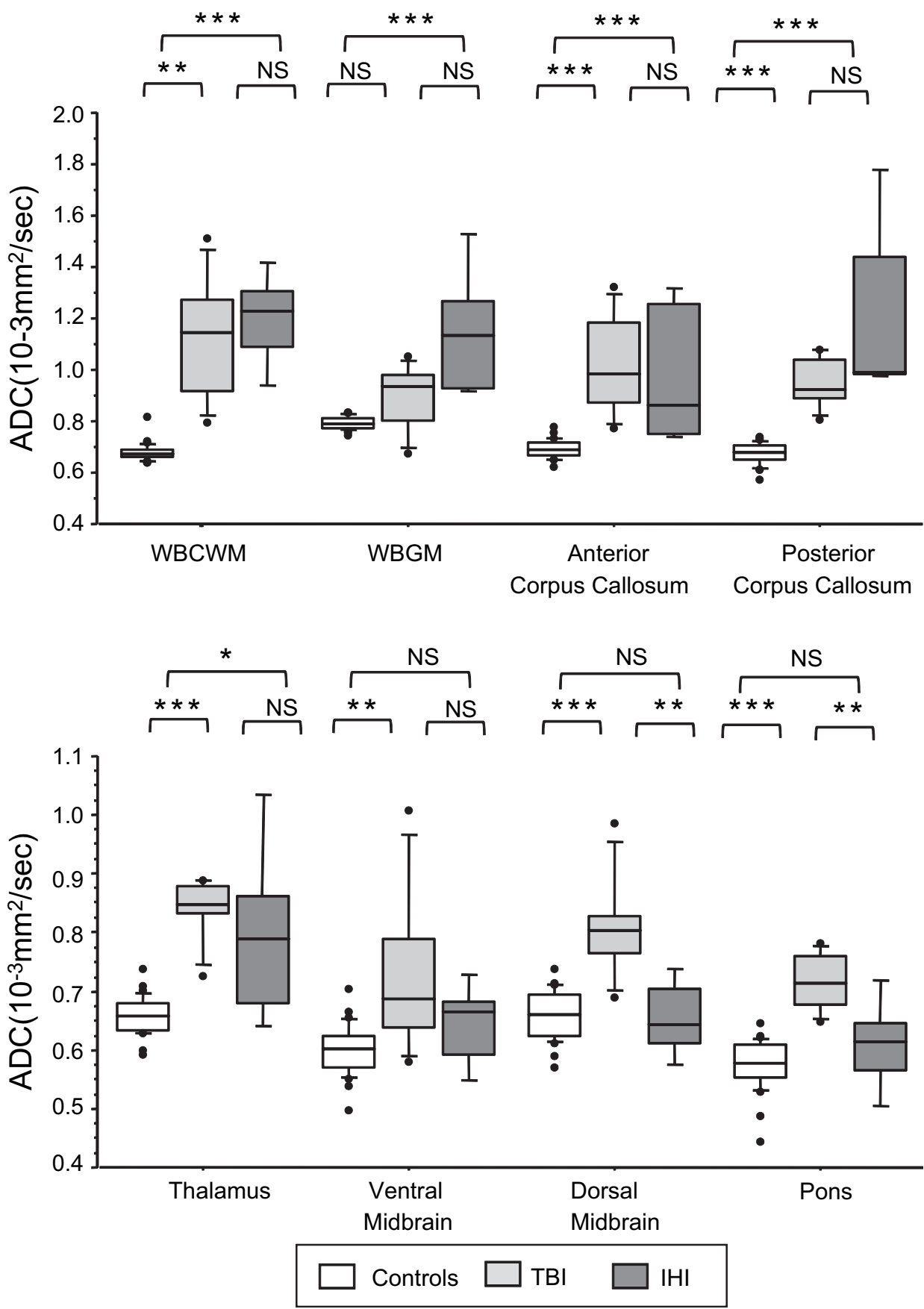

brainstem regions (dorsal and ventral midbrain and pons) which corresponded to an increase in ADC. Most of the changes in FA and $\mathrm{ADC}$ were driven by increases in radial diffusivity (table 4), although less consistent increases in axial diffusivity were noted in some brain regions, particularly in the TBI group.

In order to determine whether our measures of supratentorial white-matter damage related to preserved cortical processing, we correlated DTI parameters in the WBCWM and WBGM with clinical parameters and to fMRI responses to an auditory fMRI paradigm, graded on a three-point scale. We found significant trends of decreasing ADC in the WBGM and increasing FA in WBCWM for all patients with a higher fMRI language response (WBGM vs. $\mathrm{ADC}$ : J-T statistic $=-2.6$, $\mathrm{p}=0.009$; WBCWM vs FA: J-T statistic $=2.1, \mathrm{p}=0.033$ ). A higher CRS-R score had a positive correlation with central white matter
FA $(0.756, p=0.004)$ and inverse correlations with central ADC $(-0.871, p<0.001)$ (figure 5). These relationships were not different for the two aetiological categories. No significant correlations were found with age at scan or time from injury to scan.

\section{DISCUSSION}

We provide the first DTI comparison of the two most common etiologies of VS, namely ischaemic hypoxic brain injury and traumatic brain injury. Despite the sensitivity of conventional MRI in this setting, ${ }^{20}$ we confirm that quantitative DTI demonstrates abnormalities missed by conventional radiological assessment. Both TBI and IHI showed evidence of marked, and concordant, abnormalities in the supratentorial GM and WM compartments. These correlated with fMRI responses to 
Figure 5 Relationship of JFK modified Coma Recovery Scale (CRS-R) to the burden of supratentorial central wholebrain white matter damage (fractional anisotropy; $r=0.756(p=0.004)$, apparent diffusion coefficient; $r=-0.871(p<0.001))$. The green line represents the IQR for the controls. IHI, ischaemic/hypoxic injuries; TBI, traumatic brain injuries.
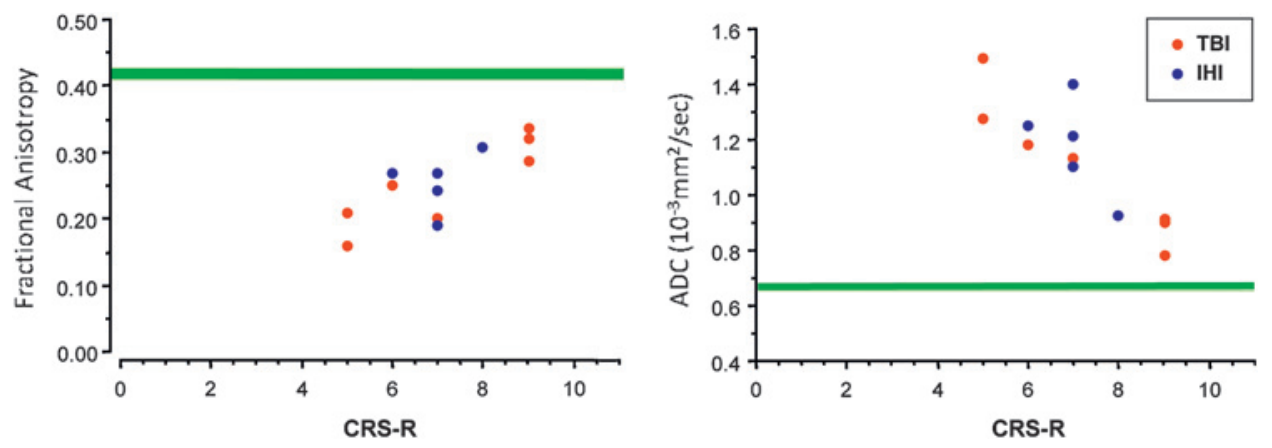

a paradigm that detected covert cortical cognitive processing, ${ }^{19}$ and to functional status as defined by the summed CRS-R. In contrast, the two aetiologies exhibited markedly discordant findings in the infratentorial compartment, where DTI abnormalities in the brainstem were confined to the TBI group. An eigenvalue analysis suggested that FA changes were consistently associated with increased radial diffusivity, with less consistent changes in axial diffusivity.

Few studies have used DTI to assess patients in chronic VS. A cross-sectional study of 52 patients with impaired consciousness (19 VS and 33 severe disability) secondary to TBI correlated motor-evoked potentials with corticospinal tract damage as found with DWI. ${ }^{21}$ One case study of a TBI patient who recovered expressive language after 19 years in the minimally conscious state (MCS) raised the intriguing possibility of DTI being able to detect neuronal plasticity, ${ }^{22}$ since this apparent functional improvement was associated with increases in anisotropy. However, the complexity of microstructural changes and the confounders caused by crossing fibres in data-processing algorithms makes it difficult to interpret these findings with confidence without larger numbers and imaging at more time points. In addition, most previous studies have classified patients based on functional status (ie, VS vs MCS) and have tended to focus on a single aetiological group. We believe that an analysis that takes account of the concordant and discordant findings in different aetiologies may provide further insights into the anatomical substrates and pathophysiology of these conditions.

The basis of VS is complex in both aetiologies. Structural and functional imaging studies, including FDG PET, have illustrated widespread damage to areas that are part of cortical networks implicated in consciousness and its constituent processes, including memory, attention and language. ${ }^{23}$ Marked structural loss has been found using both conventional MRI sequences ${ }^{24}$ and voxel-based morphometry. ${ }^{25}$ In a small sample size of five patients, with IHI VS, decreased volumes were found in the inferior parietal and superior/medial frontal cortices, insula, temporal lobes, cingulum, midbrain, dorsal pons, caudate, cerebellum and thalamus. ${ }^{25}$ FDG-PET in the same set of patients demonstrated widespread hypometabolism in the parietal and parietoccipital cortices, the cingulum, bilateral thalami, and frontal, medial and precentral gyri. 'Islands' of preserved metabolism have also been seen in some subjects, ${ }^{26}$ a finding which may help account for the small numbers of patients who, despite no other behavioural evidence of complex cortical processing, may retain some residual fragmentary verbal output. In other patients, relatively complex cognitive processing may continue, but without any behavioural evidence, and may only be detected using fMRI. ${ }^{8} 2728$ It appears, therefore, that VS may be the consequence not just of focal injury to discrete sites but also due to abnormal connectivity in distributed neuronal networks. Given this background, our demonstration of whitematter abnormalities in VS may provide an additional neuroanatomical substrate for the deficits observed. In addition, the comparison of differences in the two aetiologies may provide additional insights.

The extent of damage seen after IHI is determined by many factors which include the duration and degree of ischaemia, blood glucose levels and brain temperature during the period of insult. ${ }^{29}$ Certain brain regions exhibit a selective vulnerability to the effects of ischaemia including the cerebral cortex, striatum, hippocampus and cerebellar purkinje cells. ${ }^{12}$ Thalamic damage may be the predominant injury found at autopsy postcardiorespiratory arrest. ${ }^{30}$ The exact reasons for such increased regional susceptibility are unknown but may relate to higher metabolic rates in these regions as well as an increased proportion of excitotoxic glutaminergic cells. ${ }^{29} 31$ Our study demonstrates in vivo evidence for increased damage to particular areas including the thalamus and cerebral cortex, with the brainstem relatively preserved, which supports previous histopathological evidence ${ }^{32-35}$ as well as the widespread early (within the first 2 weeks of injury) patterns of restricted diffusion cortically, in the cerebellum and basal ganglia. ${ }^{32} 35-37$

The shearing forces experienced in TBI, and especially those with a component of traumatic axonal injury, mean that both the mechanism and the distribution of injury are different to IHI. Of course, TBI patients make also have a component of ischaemic injury, but in general the areas damaged are distinct histologically for TBI and IHI. Areas of damage in TBI are classically located in the parasagittal WM, corpus callosum (particularly the splenium/posterior aspect), and the corona radiata of the frontal and temporal lobes. ${ }^{38}$ In our study, thalamic damage was present in both patient populations, as evidenced by higher ADCs. The higher thalamic FA in hypoxic patients compared with TBI may represent the histopathological differences of predominantly neuronal loss versus retrograde degeneration, but true correlation with post-mortem studies would be required to see if this is indeed the case. In addition to abnormalities in these supratentorial regions, additional areas of damage are classically located in the dorsolateral brainstem.

These differences in the location of lesions in the two aetiologies are precisely reflected by our DTI findings. In contrast to the clear commonality of pathology in the supratentorial compartment in the two aetiologies that we studied, we found that patients with TBI exclusively demonstrated evidence of white-matter disconnection in the brainstem (figure 2). These differences underline the mechanistic heterogeneity that may result in VS. In addition, these findings may have important implications for selecting patients for interventions, or for stratification in clinical trials of novel interventions. There is 
increasing interest in using a range of therapies to enhance arousal, awareness and behavioural recovery in patients who are clinically categorised as VS. It seems self evident that not all patients are likely to respond to either pharmacological therapies or devices meant to enhance endogenous ascending neurochemical arousal systems. While functional imaging may (uniquely) provide the only evidence of preserved cortical processing in many such patients, detection of such processing may not predicate a higher likelihood of responding to therapy. Indeed, the patient who shows complex volitional control of cortical activation ${ }^{27}$ but is unable to manifest such activity through behavioural outputs may be least likely to respond to 'arousal' therapies, however intrinsically effective. Such a patient has adequate arousal; the problem is with a block in generating behavioural outputs. On the other hand, patients who show no evidence of cortical activity but have relatively preserved cortical structural integrity coupled with damage to key (brainstem) arousal systems and pathways may arguably benefit most from pharmacological ${ }^{39-42}$ or device-based enhancement ${ }^{43}$ of ascending arousal systems. This is a complex issue, and the inferences we outline above clearly remain unproven. However, these data do set novel hypotheses that can be tested. Although we have detected differences based on aetiology, findings in individual subjects, rather than aetiological groups, are the more important issue. However, the discussion above makes a case for detailed imaging of white-matter anatomy in studies that assess the impact of such therapies.

Our study has clear limitations. Despite a relatively large patient cohort in the context of previous studies, our sample size remains small, and our findings require confirmation. Further, longitudinal studies could assess the relationship between behavioural deficits and cognitive recovery on one hand, and structural and functional plasticity on the other. MRI in these patients can be challenging, and distorted anatomy may hinder traditional analysis methods-the degree of atrophy and distortion mean that careful ROI placement was required so that the chosen ROIs were easily identifiable even in abnormal brains.

Despite these limitations, the combination of conventional MRI, DTI and functional imaging may be important in this patient population. The demonstration of covert perceptual and cognitive processing using fMRI is now well established in selected patients, ${ }^{8} 2728$ but it remains unclear why only some patients exhibit such responses. The presence or absence of such functional imaging responses may be able to be explained by the distribution and/or extent of the structural damage detected using DTI techniques. In addition, as we discuss above, DTI may potentially be used to help stratify patients into those likely to respond and may allow clinicians to select subjects for more careful assessment using fMRI.

Acknowledgements We are very grateful to $\mathrm{M}$ Coleman for access to the imaging and clinical data used in this study.

Funding This research was supported by: UK National Institute of Health Research Biomedical Research Centre at Cambridge Technology Platform funding provided by the UK Department of Health. VFJN was supported by the Gates Cambridge Trust and an Overseas Research Studentship. JC and DS are supported by the NIHR Cambridge Biomedical Research Centre.

Competing interests None.

Ethics approval Ethics approval was provided by Cambridgeshire 2 Research Ethics Committee.

Patient consent Obtained.

Provenance and peer review Not commissioned; externally peer reviewed.

\section{REFERENCES}

1. Adams JH, Graham DI, Jennett B. The neuropathology of the vegetative state after an acute brain insult. Brain 2000;123(Pt 7):1327-38.

2. Adams $\mathbf{J H}$, Jennett $B$, McLellan DR, et al. The neuropathology of the vegetative state after head injury. J Clin Pathol 1999;52:804-6.

3. Kinney HC, Samuels MA. Neuropathology of the persistent vegetative state. A review. J Neuropathol Exp Neurol 1994;53:548-58.

4. Gentleman SM, Roberts GW, Gennarelli TA, et al. Axonal injury: a universal consequence of fatal closed head injury? Acta Neuropathol 1995;89:537-43.

5. Oehmichen M, Meissner C, Schmidt V, et al. Pontine axonal injury after brain trauma and nontraumatic hypoxic-ischemic brain damage. Int $J$ Legal Med 1999;112:261-7

6. Giacino JT, Kalmar K, Whyte J. The JFK Coma Recovery Scale-Revised: measurement characteristics and diagnostic utility. Arch Phys Med Rehabil 2004;85:2020-9

7. Kalmar K, Giacino JT. The JFK Coma Recovery Scale-Revised. Neuropsychol Rehabil 2005;15:454-60.

8. Coleman MR, Rodd JM, Davis MH, et al. Do vegetative patients retain aspects of language comprehension? Evidence from fMRI. Brain 2007:130(Pt 10):2494-507.

9. Coleman MR, Davis MH, Rodd JM, et al. Towards the routine use of brain imaging to aid the clinical diagnosis of disorders of consciousness. Brain 2009;132(Pt 9): 2541-52.

10. Analyze Software System. http://www.mayoresearch.mayo.edu/mayo/research/ robb lab/analyze.cfm (accessed 3 Jan 2010)

11. Holmes CJ, Hoge R, Collins L, et al. Enhancement of MR images using registration for signal averaging. J Comput Assist Tomogr 1998;22:324-33.

12. Zhang $\mathbf{Y}$, Brady M, Smith S. Segmentation of brain MR images through a hidden Markov random field model and the expectation-maximization algorithm. IEEE Trans Med Imaging 2001;20:45-57.

13. Smith SM, Jenkinson M, Johansen-Berg $\mathrm{H}$, et al. Tract-based spatial statistics: voxelwise analysis of multi-subject diffusion data. Neuroimage 2006;31:1487-505

14. FMRIB's Diffsuion Toolbox (FDT). avaliable at FSL. http://www.fmri.ox.ac.uk/fsl/ (accessed 3 Jan 2010).

15. Smith SM. Fast robust automated brain extraction. Hum Brain Mapp 2002:17:143-55

16. Vtk Cisg Registration Toolkit. http://www.image-registration.com (downloaded 2007, not currently available for download (checked January 2010).

17. Sherbondy A, Akers D, Mackenzie R, et al. Exploring connectivity of the brain's white matter with dynamic queries. IEEE Trans Vis Comput Graph 2005; 11:419-30

18. Mori S, Crain BJ, Chacko VP, et al. Three-dimensional tracking of axonal projections in the brain by magnetic resonance imaging. Ann Neurol 1999; 45:265-9.

19. Rodd JM, Davis MH, Johnsrude IS. The neural mechanisms of speech comprehension: fMRI studies of semantic ambiguity. Cereb Cortex 2005; 15:1261-9

20. Yanagawa $\mathbf{Y}$, Tsushima $Y$, Tokumaru $A$, et al. A quantitative analysis of head injury using T2* -weighted gradient-echo imaging. J Trauma 2000;49:272-7.

21. Yasokawa YT, Shinoda J, Okumura A et al. Correlation between diffusion-tensor magnetic resonance imaging and motor-evoked potential in chronic severe diffuse axonal injury. J Neurotrauma 2007;24:163-73.

22. Voss HU, Uluc AM, Dyke JP, et al. Possible axonal regrowth in late recovery from the minimally conscious state. J Clin Invest 2006;116:2005-11.

23. Giacino JT, Hirsch J, Schiff N, et al. Functional neuroimaging applications for assessment and rehabilitation planning in patients with disorders of consciousness. Arch Phys Med Rehabil 2006;87(12 Suppl 2):S67-76.

24. Ammermann $\mathbf{H}$, Kassubek J, Lotze $\mathrm{M}$, et al. MRI brain lesion patterns in patients in anoxia-induced vegetative state. J Neurol Sci 2007;260:65-70.

25. Juengling FD, Kassubek J, Huppertz HJ, et al. Separating functional and structura damage in persistent vegetative state using combined voxel-based analysis of 3-D MRI and FDG-PET. J Neurol Sci 2005;228:179-84.

26. Schiff ND, Ribary U, Moreno DR, et al. Residual cerebral activity and behavioural fragments can remain in the persistently vegetative brain. Brain 2002;125(Pt 6): 1210-34.

27. Owen AM, Coleman MR, Boly M, et al. Detecting awareness in the vegetative state. Science 2006:313:1402.

28. Owen AM, Coleman MR, Boly M, et al. Using functional magnetic resonance imaging to detect covert awareness in the vegetative state. Arch Neurol 2007:64:1098-102.

29. Auer RN, Dunn JF, Sutherland GR. Hypoxia and related conditions (Chapter 2). 8th edn. London: Edward Arnold (Publishers), 2008.

30. Kinney HC, Korein J, Panigrahy A, et al. Neuropathological findings in the brain of Karen Ann Quinlan. The role of the thalamus in the persistent vegetative state. N Eng J Med 1994;330:1469-75.

31. Greer DM. Mechanisms of injury in hypoxic-ischemic encephalopathy: implications to therapy. Semin Neurol 2006;26:373-9.

32. McKinney AM, Teksam M, Felice $R$, et al. Diffusion-weighted imaging in the setting of diffuse cortical laminar necrosis and hypoxic-ischemic encephalopathy. Am J Neuroradiol 2004:25:1659-65.

33. Kawahara H, Takeda Y, Tanaka A, et al. Does diffusion-weighted magnetic resonance imaging enable detection of early ischemic change following transient cerebral ischemia? J Neurol Sci 2000;181:73-81. 
34. Wijdicks EF, Campeau NG, Miller GM. MR imaging in comatose survivors of cardiac resuscitation. Am J Neuroradiol 2001;22:1561-5.

35. Els T, Kassubek J, Kubalek R, et al. Diffusion-weighted MRI during early global cerebral hypoxia: a predictor for clinical outcome? Acta Neurol Scand 2004;110:361-7.

36. Ward P, Counsell S, Allsop J, et al. Reduced fractional anisotropy on diffusion tensor magnetic resonance imaging after hypoxic-ischemic encephalopathy. Pediatrics 2006;117:e619-30.

37. Van Putten HP, Bouwhuis MG, Muizelaar JP, et al. Diffusion-weighted imaging of edema following traumatic brain injury in rats: effects of secondary hypoxia. $J$ Neurotrauma 2005;22:857-72.

38. Kampfl A, Franz G, Aichner F, et al. The persistent vegetative state after closed head injury: clinical and magnetic resonance imaging findings in 42 patients. J Neurosurg 1998;88:809-16.
39. Kim YH, Ko MH, Na SY, et al. Effects of single-dose methylphenidate on cognitive performance in patients with traumatic brain injury: a double-blind placebo-controlled study. Clin Rehabil 2006;20:24-30.

40. Leonard BE, McCartan D, White J, et al. Methylphenidate: a review of its neuropharmacological, neuropsychological and adverse clinical effects. Hum Psychopharmacol 2004:19:151-80.

41. Pavlovskaya M, Hochstein S, Keren 0, et al. Methylphenidate effect on hemispheric attentional imbalance in patients with traumatic brain injury: a psychophysical study. Brain Inj 2007;21:489-97.

42. Teitelman E. Off-label uses of modafinil. Am J Psychiatry 2001;158:1341.

43. Schiff ND, Giacino JT, Kalmar K, et al. Behavioural improvements with thalamic stimulation after severe traumatic brain injury. Nature 2007:448: 600-3. 